\section{Intermediate density lipoprotein}

\section{K. J. Lackner ${ }^{1}$ und D. Peetz ${ }^{2}$}

${ }^{1}$ Institut für Klinische Chemie und Laboratoriumsmedizin, Universitätsmedizin Mainz, Mainz, Deutschland

${ }^{2}$ Institut für Labormedizin, Helios Klinikum Berlin-Buch, Berlin, Deutschland

$\operatorname{Synonym(e)~IDL~}$

Englischer Begriff intermediate density lipoprotein
Definition Lipoproteine mit einer hydratisierten Dichte von $1,006-1,019 \mathrm{~g} / \mathrm{mL}$

Beschreibung IDL sind eine kleine Lipoproteinfraktion, die in Dichte, Größe, Lipid- und Proteinzusammensetzung zwischen $>$ Very low density Lipoprotein (VLDL) und > Low density lipoprotein (LDL) liegen. Sie entstehen im Stoffwechsel der plasmatischen Lipoproteine aus VLDL hauptsächlich durch die Einwirkung der Lipoproteinlipase und stellen im Prinzip ein VLDL-Remnant-Partikel dar. IDL werden weiter umgebaut zu LDL. In der konventionellen Diagnostik werden sie meist unter die LDL-Fraktion subsumiert, auch wenn dies nicht ganz korrekt ist. 